ДОПОЛНИТЕЛЬНЫЕ МАТЕРИАЛЫ К СТАТЬЕ

\title{
АНАЛИЗ РЕНТГЕНОСТРУКТУРНЫХ ХАРАКТЕРИСТИК ГЛИКОЛУРИЛА И ЕГО ПРОИЗВОДНЫХ
}

\author{
С.Ю. Паньшина ${ }^{1,2}$, О.В. Пономаренко ${ }^{3}$, А.А. Бакибаев ${ }^{1}$, В.С. Мальков ${ }^{1}$ \\ ${ }^{1}$ Томский государственный университет, Томск, Россия \\ ${ }^{2}$ Томский политехнический университет, Томск, Россия \\ E-mail: janim_svetatusik@mail.ru \\ ${ }^{3}$ Евразийский национальный университет им. Л.Н. Гумилева, Нур-Султан, Республика Казахстан
}

\section{Таблицы}

Таблица Д1 - Водородные связи в гликолурилах 3 и 4

\begin{tabular}{|c|c|c|c|c|c|}
\hline \multirow{2}{*}{$№$} & \multirow{2}{*}{ Связи } & \multicolumn{3}{|c|}{ Длины связей, } & угол, град \\
\cline { 3 - 6 } & & $\mathrm{D}-\mathrm{H}$ & $\mathrm{H} \ldots \mathrm{A}$ & $\mathrm{D} \ldots \mathrm{A}$ & DHА \\
\hline \multirow{3}{*}{$\mathbf{3}$} & $\mathrm{N}_{4}-\mathrm{H} \ldots \mathrm{O}_{1}$ & $0.75(6)$ & $2.14(6)$ & $2.88(1)$ & 165.6 \\
\hline & $\mathrm{N}_{4 \mathrm{a}}-\mathrm{H} \ldots \mathrm{O}_{1 \mathrm{a}}$ & $0.75(3)$ & $2.17(3)$ & $2.812(5)$ & 169.6 \\
& $\mathrm{~N}_{6 \mathrm{a}}-\mathrm{H} \ldots \mathrm{O}_{2 \mathrm{~b}}$ & $0.86(4)$ & $2.08(4)$ & $2.890(5)$ & 158.3 \\
& $\mathrm{~N}_{4 \mathrm{~b}}-\mathrm{H} \ldots \mathrm{O}_{1 \mathrm{~b}}$ & $0.77(3)$ & $2.12(3)$ & $2.892(4)$ & 173.3 \\
& $\mathrm{~N}_{6 \mathrm{~b}}-\mathrm{H} \ldots \mathrm{O}_{1 \mathrm{a}}$ & $0.80(4)$ & $1.96(4)$ & $2.837(5)$ & 162.4 \\
\hline
\end{tabular}

Таблица Д2 - Расстояния между металлическими центрами и валентные углы комплексов 15a-e

\begin{tabular}{|c|c|c|c|c|c|c|}
\hline \multirow{2}{*}{ № } & \multirow{2}{*}{$\begin{array}{c}\text { Координационное } \\
\text { число }\end{array}$} & \multicolumn{2}{|c|}{ Расстояние, $\AA$} & \multicolumn{2}{|c|}{ Валентные углы, град } \\
\cline { 3 - 7 } & 7 & $\mathrm{Me} . . \mathrm{Me}$ & $\mathrm{Me} . . \mathrm{O}$ & $1 . \mathrm{MeOC}$ & $2 . \mathrm{MeOC}$ & ОМеО \\
\hline $\mathbf{1 5 a}$ & 10 & $8.7261(9)$ & $2.149(2)$ & $131.37(2)$ & $157.75(2)$ & $85.16(7)$ \\
\hline \multirow{2}{*}{$\mathbf{1 5 b}$} & 10 & $9.8726(7)$ & $\begin{array}{c}2.5502(18) \text { и } \\
2.5012(18)\end{array}$ & $144.04(1)$ & $166.98(2)$ & $69.96(6)$ \\
\hline $\mathbf{1 5 c}$ & 10 & $9.7904(4)$ & $2.506(1)$ и 2.460(2) & $143.51(1)$ & $166.47(1)$ & $70.29(5)$ \\
\hline $\mathbf{1 5 d}$ & 9 & $6.8697(4)$ & $2.3671(14)$ & $136.54(1)$ & $132.88(1)$ & $152.52(5)$ \\
\hline $\mathbf{1 5 e}$ & \multicolumn{5}{|c|}{}
\end{tabular}

Таблица Д3 - Геометрические параметры водородных связей в структурах 15a-e

\begin{tabular}{|c|c|c|c|c|c|c|c|c|c|c|c|}
\hline \multirow{2}{*}{ № } & \multirow{2}{*}{ Связь } & \multicolumn{3}{|c|}{ Расстояние, $\AA$} & \multirow{2}{*}{\begin{tabular}{|c|} 
Угол, град \\
D-H...A
\end{tabular}} & \multirow{2}{*}{ № } & \multirow{2}{*}{ Связь } & \multicolumn{3}{|c|}{ Расстояние, $\AA$} & \multirow{2}{*}{\begin{tabular}{|c|} 
Угол, град \\
D-H...A \\
\end{tabular}} \\
\hline & & D-H & H...A & D...A & & & & D-H & H...A & D...A & \\
\hline \multirow{7}{*}{$15 \mathbf{a}$} & $\mathrm{C}_{6}-\mathrm{H}_{9} \ldots \mathrm{O}$ & $0.93(18)$ & $2.45(18)$ & $3.375(3)$ & 177.(2) & \multirow{3}{*}{$15 c$} & $\mathrm{O}_{\mathrm{w}}-\mathrm{H}_{15} \ldots \mathrm{O}_{4}$ & $0.80(3)$ & $2.16(3)$ & $2.902(17)$ & 154.(3) \\
\hline & $\mathrm{C}_{6}-\mathrm{H}_{10} \ldots \mathrm{O}$ & $0.93(18)$ & $2.66(2)$ & $3.519(3)$ & 155.(3) & & $\mathrm{O}_{\mathrm{w}}-\mathrm{H}_{17} \ldots \mathrm{O}_{2}$ & $0.75(3)$ & 2.03(3) & $2.734(19)$ & 156.(3) \\
\hline & $\mathrm{O}_{9}-\mathrm{H}_{15} \ldots \mathrm{O}$ & $0.81(4)$ & $2.14(4)$ & 2.934(3) & 166.(3) & & $\mathrm{O}_{\mathrm{w}}-\mathrm{H}_{18} \ldots \mathrm{O}_{8}$ & $0.77(3)$ & $2.38(3)$ & $3.098(2)$ & 156.(3) \\
\hline & $\mathrm{O}_{9}-\mathrm{H}_{6} \ldots \mathrm{O}$ & $0.83(4)$ & $1.85(4)$ & $2.677(4)$ & 171.(4) & \multirow{4}{*}{$15 d$} & $\mathrm{O}_{w}-\mathrm{H}_{1} \ldots \mathrm{O}_{7}$ & $0.85(2)$ & $2.22(2)$ & $2.911(3)$ & 139.(3) \\
\hline & $\mathrm{O}_{10}-\mathrm{H}_{18} \ldots \mathrm{O}$ & $0.83(10)$ & $2.19(14)$ & $3.007(3)$ & 164.(4) & & $\mathrm{O}_{w}-\mathrm{H}_{2} \ldots \mathrm{O}_{4}$ & $0.85(2)$ & $2.14(17)$ & $2.899(3)$ & 149.(3) \\
\hline & $\mathrm{O}_{10}-\mathrm{H}_{17} \ldots \mathrm{N}_{2}$ & \begin{tabular}{|l|}
$0.84(10)$ \\
\end{tabular} & $2.68(14)$ & $3.504(3)$ & 169.(4) & & $\mathrm{O}_{w}-\mathrm{H}_{3} \ldots \mathrm{O}_{2}$ & $0.85(2)$ & $1.94(12)$ & $2.731(3)$ & 154.(2) \\
\hline & $\mathrm{O}_{10}-\mathrm{H}_{17} \ldots \mathrm{O}_{5}$ & $\begin{array}{l}0.84(10) \\
\end{array}$ & $2.30(18)$ & $3.100(3)$ & $160 .(4)$ & & $\mathrm{O}_{\mathrm{w}}-\mathrm{H}_{1} \ldots \mathrm{O}_{11}$ & $0.85(2)$ & $2.23(12)$ & $3.059(3)$ & $166 .(4)$ \\
\hline \multirow{5}{*}{$15 b$} & $\mathrm{O}_{11}-\mathrm{H}_{1} \ldots \mathrm{O}_{3}$ & $0.73(4)$ & $2.03(4)$ & $2.727(4)$ & 163.(3) & \multirow{5}{*}{$15 \mathrm{e}$} & $\mathrm{C}_{6}-\mathrm{H}_{4} \ldots \mathrm{O}_{9}$ & $0.93(3)$ & $2.63(3)$ & $3.059(3)$ & 169.(2) \\
\hline & $\mathrm{O}_{11}-\mathrm{H}_{2} \ldots \mathrm{O}_{4}$ & $0.82(5)$ & $2.42(5)$ & $3.220(3)$ & 168.(4) & & $\mathrm{C}_{8}-\mathrm{H}_{10} \ldots \mathrm{O}_{10}$ & $0.95(3)$ & $2.62(3)$ & 3.059(3) & 157.(2) \\
\hline & $\mathrm{O}_{10}-\mathrm{H}_{3} \ldots \mathrm{O}_{7}$ & $0.77(4)$ & $2.19(4)$ & $2.902(3)$ & 154.(4) & & $\mathrm{O}_{12}-\mathrm{H}_{15} \ldots \mathrm{O}_{1}$ & $0.92(3)$ & $1.78(3)$ & $3.059(3)$ & 173.(3) \\
\hline & $\mathrm{O}_{10}-\mathrm{H}_{4} \ldots \mathrm{O}_{6}$ & $0.71(4)$ & $2.30(5)$ & $2.936(3)$ & $152 .(5)$ & & $\mathrm{O}_{12}-\mathrm{H}_{16} \ldots \mathrm{O}_{3}$ & $0.74(3)$ & $2.17(3)$ & $2.920(3)$ & 178.(3) \\
\hline & & & & & & & $\mathrm{O}_{12}-\mathrm{H}_{16} \ldots \mathrm{O}_{4}$ & $0.93(3)$ & $2.64(3)$ & $2.920(3)$ & 128.(3) \\
\hline
\end{tabular}

(C) Паньшина С.Ю., Пономаренко О.В., Бакибаев А.А., Мальков В.С., 2020 
Таблица Д4 - Геометрические параметры комплексов мебикара 15a-e

\begin{tabular}{|c|c|c|c|c|c|}
\hline \multirow{2}{*}{$\begin{array}{c}\text { Связи, } \\
\AA\end{array}$} & \multicolumn{5}{|c|}{ Комплексы } \\
\cline { 2 - 6 } & $\mathbf{1 5 a}$ & $\mathbf{1 5 b}$ & $\mathbf{1 5 c}$ & $\mathbf{1 5 d}$ & $\mathbf{1 5 e}$ \\
\hline $\mathrm{O}_{1}-\mathrm{C}_{3}$ & 1.23 & 1.24 & 1.24 & 1.24 & 1.24 \\
\hline $\mathrm{O}_{2}-\mathrm{C}_{7}$ & 1.24 & 1.25 & 1.24 & 1.25 & 1.25 \\
\hline $\mathrm{C}_{1}-\mathrm{C}_{5}$ & 1.55 & 1.54 & 1.55 & 1.55 & 1.55 \\
\hline $\mathrm{N}_{2}-\mathrm{C}_{1}$ & 1.44 & 1.45 & 1.45 & 1.45 & 1.45 \\
\hline $\mathrm{N}_{2}-\mathrm{C}_{3}$ & 1.34 & 1.35 & 1.35 & 1.35 & 1.35 \\
\hline $\mathrm{N}_{4}-\mathrm{C}_{3}$ & 1.35 & 1.35 & 1.35 & 1.34 & 1.35 \\
\hline $\mathrm{N}_{4}-\mathrm{C}_{5}$ & 1.44 & 1.45 & 1.45 & 1.45 & 1.45 \\
\hline $\mathrm{N}_{6}-\mathrm{C}_{5}$ & 1.44 & 1.45 & 1.45 & 1.44 & 1.45 \\
\hline $\mathrm{N}_{6}-\mathrm{C}_{7}$ & 1.34 & 1.35 & 1.35 & 1.35 & 1.35 \\
\hline $\mathrm{N}_{8}-\mathrm{C}_{1}$ & 1.44 & 1.5 & 1.45 & 1.45 & 1.45 \\
\hline $\mathrm{N}_{8}-\mathrm{C}_{7}$ & 1.35 & 1.35 & 1.35 & 1.35 & 1.34 \\
\hline $\begin{array}{c}\text { Диэд- } \\
\text { ральный } \\
\text { угол, }\end{array}$ & 120.3 & 124 & 123.9 & 123.7 & 117.1 \\
град. & & & & & \\
\hline
\end{tabular}

Таблица Д5 - Значение длин связей для гликолурила 1 и N-алкилзамещенных гликолурилов 2-14, 16 и металлических комплексов 3a, 6а и 15a-e

\begin{tabular}{|c|c|c|c|c|c|c|c|c|c|c|c|c|c|c|c|c|c|c|c|c|c|c|}
\hline $\begin{array}{c}\text { Связи } \\
, \AA\end{array}$ & 1 & 2 & 3 & 3a & 4 & 5 & 6 & $6 a$ & 7 & 8 & 9 & 10 & 11 & 12 & 13 & 14 & $15 a$ & $15 b$ & $15 c$ & $15 d$ & $15 e$ & 16 \\
\hline $\mathrm{O}_{1}-\mathrm{C}_{3}$ & 24 & & 21 & 24 & 3 & 23 & 22 & \begin{tabular}{|l|}
1.24 \\
\end{tabular} & 23 & 23 & 1.23 & 24 & 23 & 1.23 & 1.23 & 22 & 23 & 24 & 24 & 24 & 24 & .24 \\
\hline $\mathrm{O}_{2}-\mathrm{C}_{7}$ & 24 & 24 & 21 & 24 & 1.23 & 23 & .24 & 1.24 & 1.23 & 23 & 1.23 & 1.24 & 23 & 1.24 & 1.22 & .23 & 1.24 & 1.25 & .24 & 1.25 & 1.25 & .23 \\
\hline $\mathrm{C}_{1}-\mathrm{C}_{5}$ & 56 & 56 & 55 & 1.56 & 1.55 & 1.54 & 1.50 & \begin{tabular}{|l|}
1.56 \\
\end{tabular} & 1.55 & 1.55 & 1.55 & \begin{tabular}{|l|}
1.57 \\
\end{tabular} & .55 & 1.55 & 1.55 & 1.55 & 1.55 & \begin{tabular}{|l|}
1.54 \\
\end{tabular} & 1.55 & \begin{tabular}{|l|}
1.55 \\
\end{tabular} & \begin{tabular}{|l|}
1.55 \\
\end{tabular} & \begin{tabular}{|l|}
1.54 \\
\end{tabular} \\
\hline $\mathrm{N}_{2}-\mathrm{C}_{1}$ & 44 & 44 & 45 & 44 & 1.45 & 44 & 44 & \begin{tabular}{|l|}
1.44 \\
\end{tabular} & 1.43 & 44 & 1.45 & \begin{tabular}{|l|}
1.46 \\
\end{tabular} & 44 & 44 & 44 & .44 & 44 & 1.45 & .45 & .45 & 1.45 & \begin{tabular}{|l|}
1.43 \\
\end{tabular} \\
\hline $\mathrm{N}_{2}-\mathrm{C}_{3}$ & 35 & .36 & 36 & .37 & 1.35 & 1.36 & 1.35 & 1.35 & 1.33 & 1.36 & 1.37 & 1.37 & 1.37 & 35 & 1.36 & 1.37 & 1.34 & 1.35 & 1.35 & 1.35 & 1.35 & .36 \\
\hline $\mathrm{N}_{4}-\mathrm{C}_{3}$ & 35 & 36 & 36 & 1.34 & 1.35 & 1.36 & 1.35 & 1.36 & 1.31 & 1.37 & 1.38 & \begin{tabular}{|l|}
1.36 \\
\end{tabular} & 1.39 & & 37 & \begin{tabular}{|l|}
1.37 \\
\end{tabular} & 1.35 & 1.35 & 1.35 & \begin{tabular}{|l|}
1.34 \\
\end{tabular} & 1.35 & \begin{tabular}{|l|}
1.34 \\
\end{tabular} \\
\hline $\mathrm{N}_{4}-\mathrm{C}_{5}$ & 44 & 45 & 45 & .45 & 1.43 & 1.45 & \begin{tabular}{|l|}
1.44 \\
\end{tabular} & \begin{tabular}{|l|}
1.46 \\
\end{tabular} & 1.48 & 1.45 & 1.46 & \begin{tabular}{|l|}
1.44 \\
\end{tabular} & 1.45 & 1.45 & 1.46 & 1.44 & 1.44 & 1.45 & 1.45 & 1.45 & \begin{tabular}{|l|}
1.45 \\
\end{tabular} & 1.45 \\
\hline $\mathrm{N}_{6}-\mathrm{C}_{5}$ & 44 & .44 & 45 & .42 & 1.43 & 1.46 & 1.44 & 1.44 & 1.44 & 1.45 & 1.44 & 1.45 & 1.44 & 1.44 & 1.44 & 1.45 & 1.44 & 1.45 & 1.45 & 1.44 & 1.45 & 1.43 \\
\hline $\mathrm{N}_{6}-\mathrm{C}_{7}$ & 35 & 36 & 1.36 & .36 & 1.35 & 1.36 & \begin{tabular}{|l|}
1.37 \\
\end{tabular} & 1.35 & 1.38 & \begin{tabular}{|l|}
1.37 \\
\end{tabular} & 1.37 & 1.35 & 1.36 & 1.35 & 1 & \begin{tabular}{|l|}
1.37 \\
\end{tabular} & 1.34 & 1.35 & 1.35 & 1.35 & 1.35 & 1.36 \\
\hline $\mathrm{N}_{8}-\mathrm{C}_{1}$ & 44 & \begin{tabular}{|l|}
1.45 \\
\end{tabular} & 1.45 & 1.43 & 1.45 & 1.45 & \begin{tabular}{|l|}
1.44 \\
\end{tabular} & \begin{tabular}{|l|}
1.46 \\
\end{tabular} & 1.45 & 1.45 & 1.45 & 1.45 & 1.45 & 1.44 & 1.45 & 1.44 & 1.44 & 1.50 & 1.45 & \begin{tabular}{|l|}
1.45 \\
\end{tabular} & \begin{tabular}{|l|}
1.45 \\
\end{tabular} & 1.45 \\
\hline $\mathrm{N}_{8}-\mathrm{C}_{7}$ & 1.35 & 1.36 & 1.36 & 1.36 & 1.35 & 1.37 & 1.37 & 1.35 & 1.36 & 1.36 & 1.37 & 1.36 & 1.35 & 1.35 & 1.37 & 1.35 & 1.35 & 1.35 & 1.35 & 1.35 & 1.34 & 1.35 \\
\hline $2-\mathrm{C}_{10}$ & - & - & 1.44 & 1.48 & - & 1.47 & 1.45 & 1.46 & 1.47 & 1.44 & 1.47 & 1.49 & 1.45 & & & & & & - & & - & - \\
\hline $\mathrm{N}_{4}-\mathrm{C}_{9}$ & - & .44 & $\pi$ & - & 1.46 & 1.43 & \begin{tabular}{|l|}
1.46 \\
\end{tabular} & \begin{tabular}{|l|}
1.46 \\
\end{tabular} & 1.45 & \begin{tabular}{|l|}
1.44 \\
\end{tabular} & 1.45 & - & - & 44 & 1.44 & 1.46 & & & - & 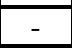 & - & 1.44 \\
\hline $\mathrm{N}_{6}-\mathrm{C}_{8}$ & - & 1.45 & 1.44 & 1.46 & 1.46 & 1.46 & 1.45 & - & 1.45 & 1.47 & 1.47 & - & - & & 1.45 & 1.45 & - & - & - & - & - & - \\
\hline $\mathrm{N}_{8}-\mathrm{C}_{11}$ & - & - & $=$ & - & 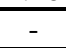 & 1.43 & \begin{tabular}{|l|}
1.46 \\
\end{tabular} & - & - & \begin{tabular}{|l|}
1.45 \\
\end{tabular} & 1.45 & & & & 1.45 & \begin{tabular}{|l|}
1.45 \\
\end{tabular} & & & & & - & 1.43 \\
\hline
\end{tabular}

Таблица Д6 - Значение углов в градусах для $\mathrm{N}$-алкилзамещенных соединений 1-10, 12-16 и металлических комплексов 3a, 6а

\begin{tabular}{|c|c|c|c|c|c|c|c|c|c|c|c|c|c|c|c|c|}
\hline Углы, град & 1 & 2 & 3 & $3 \mathbf{a}$ & 4 & 5 & 6 & $6 a$ & 7 & 8 & 9 & 10 & 12 & 13 & 14 & 16 \\
\hline $\mathrm{C}_{1}-\mathrm{N}_{2}-\mathrm{C}_{3}$ & 112.6 & 113.1 & 112.9 & 111.3 & 112.5 & 112.7 & 111.0 & 111.4 & 112.5 & 122.7 & 112.3 & 112.3 & 112.5 & 113.1 & 111.7 & 111.8 \\
\hline $\mathrm{C}_{3}-\mathrm{N}_{4}-\mathrm{C}_{5}$ & 112.6 & 111.9 & 113.5 & 112.7 & 113.6 & 111.5 & 113.0 & 111.7 & 111.2 & 112.6 & 112.5 & 111.3 & 112.1 & 112.7 & 112.6 & 112.1 \\
\hline $\mathrm{C}_{5}-\mathrm{N}_{6}-\mathrm{C}_{7}$ & 112.6 & 112.2 & 112.9 & 112.6 & 113.6 & 111.7 & 111.0 & 111.8 & 108.8 & 111.6 & 112.0 & 113.4 & 112.2 & 113.1 & 111.9 & 112.5 \\
\hline $\mathrm{C}_{1}-\mathrm{N}_{8}-\mathrm{C}_{7}$ & 112.6 & 112.5 & 113.5 & 112.6 & 112.5 & 110.9 & 110.0 & 111.9 & 112.6 & 112.9 & 111.6 & 112.1 & 112.6 & 111.7 & 113.2 & 112.5 \\
\hline $\mathrm{N}_{2}-\mathrm{C}_{1}-\mathrm{C}_{5}$ & 102.8 & 102.7 & 103.1 & 104.2 & 103.1 & 113.3 & 113.0 & - & 111.7 & 103.4 & 103.5 & 102.8 & 102.9 & 103.5 & 103.6 & 103.5 \\
\hline $\mathrm{N}_{2}-\mathrm{C}_{1}-\mathrm{N}_{8}$ & 114.7 & 114.8 & 113.8 & 113.2 & 114.6 & 113.3 & 113.6 & - & 115.5 & 114.9 & 114.8 & 114.6 & 115.1 & 112.9 & 113.8 & 114.3 \\
\hline $\mathrm{N}_{2}-\mathrm{C}_{3}-\mathrm{N}_{4}$ & 109.1 & 108.6 & 107.5 & 109.4 & 108.3 & 108.6 & 107.9 & - & 110.0 & 108.1 & 107.9 & 109.6 & 108.7 & 108.1 & 108.2 & 109.1 \\
\hline $\mathrm{N}_{4}-\mathrm{C}_{5}-\mathrm{C}_{1}$ & 102.8 & 103.5 & 102.7 & 101.7 & 102.7 & 104.0 & 104.0 & - & 102.2 & 102.6 & 102.3 & 103.3 & 103.1 & 102.4 & \begin{tabular}{ll|}
103.0 \\
\end{tabular} & 114.3 \\
\hline $\mathrm{N}_{4}-\mathrm{C}_{5}-\mathrm{N}_{6}$ & 114.7 & 114.6 & 113.8 & 115.0 & 114.4 & 114.4 & 114.5 & - & 113.6 & 114.4 & 114.4 & 114.7 & 114.4 & 115.1 & 114.6 & 114.0 \\
\hline $\mathrm{N}_{6}-\mathrm{C}_{5}-\mathrm{C}_{1}$ & 102.8 & 103.4 & 103.1 & 103.2 & 102.7 & 103.8 & 104.0 & - & 105.4 & 104.1 & 103.6 & 103.0 & 103.2 & 103.2 & 103.0 & 103.4 \\
\hline $\mathrm{N}_{6}-\mathrm{C}_{7}-\mathrm{N}_{8}$ & 109.1 & 109.1 & 107.5 & 108.5 & 108.3 & 109.8 & 107.9 & - & 110.6 & 108.5 & 108.3 & 108.9 & 108.5 & 108.2 & 108.7 & 108.4 \\
\hline $\mathrm{N}_{8}-\mathrm{C}_{1}-\mathrm{C}_{5}$ & 102.8 & 102.6 & 102.7 & 102.5 & 103.1 & 104.5 & 103.3 & - & 103.8 & 102.3 & 102.5 & 103.0 & 102.7 & 103.5 & 103.0 & 102.3 \\
\hline $\mathrm{O}_{1}-\mathrm{C}_{3}-\mathrm{N}_{4}$ & 125.5 & 125.3 & 125.6 & 124.2 & 125.9 & 125.2 & 126.7 & - & 125.4 & 126.2 & 125.6 & 126.4 & 125.3 & 125.1 & 125.3 & 125.5 \\
\hline $\mathrm{O}_{1}-\mathrm{C}_{3}-\mathrm{N}_{2}$ & 125.5 & 126.0 & 126.7 & 126.3 & 125.8 & 126.2 & 125.4 & - & 124.4 & 125.8 & 126.5 & 124.0 & 126.0 & 1268 & 126.5 & 125.4 \\
\hline $\mathrm{O}_{2}-\mathrm{C}_{7}-\mathrm{N}_{8}$ & 125.5 & 125.4 & 125.6 & 124.6 & - & 125.3 & - & - & 127.2 & 125.3 & 125.3 & 125.9 & 126.1 & 125.4 & 126.5 & 125.6 \\
\hline $\mathrm{O}_{2}-\mathrm{C}_{7}-\mathrm{N}_{6}$ & 125.5 & 125.5 & 126.7 & 126.8 & - & 124.9 & 124.6 & - & 122.2 & 126.2 & 126.3 & 125.2 & 125.4 & 126.4 & 124.9 & 126.0 \\
\hline $\mathrm{C}_{1}-\mathrm{N}_{8}-\mathrm{C}_{11}$ & - & - & - & 124.0 & - & 124.8 & 123.9 & 123.2 & & 123.1 & 121.8 & - & & 107.5 & 122.5 & 123.1 \\
\hline
\end{tabular}


ЖУРНАЛ СТРУКТУРНОЙ ХИМИИ. 2020. Т. 61, № 9

\begin{tabular}{|c|c|c|c|c|c|c|c|c|c|c|c|c|c|c|c|c|}
\hline $\mathrm{C}_{1}-\mathrm{N}_{2}-\mathrm{C}_{10}$ & - & - & - & - & 124.4 & $112.7 \mid$ & 123.4 & 125.6 & 119.2 & 124.2 & 122.6 & - & & & & - \\
\hline $\mathrm{C}_{3}-\mathrm{N}_{2}-\mathrm{C}_{10}$ & - & - & - & 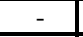 & 122.1 & 121.4 & 121.6 & 122.6 & 128.2 & 123.1 & 126.5 & - & & & & - \\
\hline $\mathrm{C}_{3}-\mathrm{N}_{4}-\mathrm{C}_{9}$ & - & 122.8 & - & 121.6 & - & 124.2 & 121.0 & 121.0 & 126.0 & 123.4 & 122.7 & 123.2 & 122.1 & 122.0 & 120.7 & 124.9 \\
\hline $\mathrm{C}_{5}-\mathrm{N}_{4}-\mathrm{C}_{9}$ & - & 123.3 & & 122.4 & - & 124.2 & 123.4 & 124.3 & 122.0 & 123.2 & 122.8 & 125.4 & 123.6 & 123.2 & 124.9 & 122.9 \\
\hline $\mathrm{C}_{5}-\mathrm{N}_{6}-\mathrm{C}_{8}$ & - & 123.3 & 121.4 & - & - & 111.7 & 123.9 & 127.9 & 124.4 & 122.0 & 123.0 & - & & 123.4 & 122.5 & - \\
\hline $\mathrm{C}_{7}-\mathrm{N}_{6}-\mathrm{C}_{8}$ & - & 123.7 & 124.5 & - & - & 122.2 & 125.2 & 120.1 & 125.6 & 126.2 & 124.0 & - & & 123.5 & 122.1 & - \\
\hline $\mathrm{C}_{7}-\mathrm{N}_{8}-\mathrm{C}_{11}$ & - & - & - & 122.2 & - & 124.8 & 121.1 & 124.7 & - & 123.1 & 120.8 & - & & 114.8 & 124.7 & 124.3 \\
\hline $\begin{array}{r}\text { Угол между } \\
\text { плоскостями }\end{array}$ & 124.1 & 121.4 & 121.0 & 120.4 & 119.0 & 118.9 & 119.1 & 119.0 & 118.5 & 120.5 & 121.8 & 122.4 & 125.5 & 120.9 & 121.9 & 118.7 \\
\hline
\end{tabular}

Таблица Д7 - Формирование водородных связей молекул 17a и $17 \mathbf{b .}$

\begin{tabular}{|c|c|c|c|c|c|c|c|c|c|}
\hline \multirow{2}{*}{$\begin{array}{c}\mathbf{1 7 a} \\
\text { Связь }\end{array}$} & \multicolumn{3}{|c|}{ Расстояние, $\AA$} & \multirow{2}{*}{$\begin{array}{c}\begin{array}{c}\text { Угол, } \\
\text { град }\end{array} \\
\begin{array}{c}\text { D- } \\
\text { H...A }\end{array}\end{array}$} & \multirow{2}{*}{$\begin{array}{c}\mathbf{1 7 b} \\
\text { Связь }\end{array}$} & \multicolumn{3}{|c|}{ Расстояние, А̊ } & \multirow{2}{*}{\begin{tabular}{|c|} 
Угол, град \\
D-H...A
\end{tabular}} \\
\hline & D-H & H...A & D...A & & & D-H & H..A & D..A & \\
\hline $\mathrm{N}_{6}-\mathrm{H} \ldots \mathrm{O}_{1}$ & 0.89 & 2.03 & 2.91 & 173.3 & $\mathrm{~N}_{6}-\mathrm{H} \ldots \mathrm{O}_{1}$ & 0.85 & 2.08 & 2.94 & 167.2 \\
\hline $\mathrm{N}_{8}-\mathrm{H} \ldots \mathrm{O}_{2}$ & 0.87 & 2.06 & 2.93 & 170.6 & $\mathrm{~N}_{8}-\mathrm{H} \ldots \mathrm{O}_{2}$ & 0.87 & 2.12 & 2.99 & 170.0 \\
\hline
\end{tabular}

Таблица Д8 - Значение длин связей для гликолурила 1 и его N-фенил-, арильных производных 17-19

\begin{tabular}{|c|c|c|c|c|c|}
\hline $\begin{array}{c}\text { Связи, } \\
\mathbf{\AA}\end{array}$ & $\mathbf{1}$ & $\mathbf{1 7 a}$ & $\mathbf{1 7 b}$ & $\mathbf{1 8}$ & $\mathbf{1 9}$ \\
\hline $\mathrm{O}_{1}-\mathrm{C}_{3}$ & 1.24 & 1.24 & 1.24 & 1.22 & 1.24 \\
\hline $\mathrm{O}_{2}-\mathrm{C}_{7}$ & 1.24 & 1.23 & 1.24 & 1.22 & 1.25 \\
\hline $\mathrm{C}_{1}-\mathrm{C}_{5}$ & 1.56 & 1.56 & 1.55 & 1.56 & 1.57 \\
\hline $\mathrm{N}_{2}-\mathrm{C}_{1}$ & 1.44 & 1.45 & 1.44 & 1.45 & 1.44 \\
\hline $\mathrm{N}_{2}-\mathrm{C}_{3}$ & 1.35 & 1.36 & 1.36 & 1.38 & 1.36 \\
\hline $\mathrm{N}_{4}-\mathrm{C}_{3}$ & 1.35 & 1.36 & 1.36 & 1.38 & 1.36 \\
\hline $\mathrm{N}_{4}-\mathrm{C}_{5}$ & 1.44 & 1.46 & 1.46 & 1.44 & 1.46 \\
\hline $\mathrm{N}_{6}-\mathrm{C}_{5}$ & 1.44 & 1.45 & .44 & 1.45 & 1.44 \\
\hline $\mathrm{N}_{6}-\mathrm{C}_{7}$ & 1.35 & 1.37 & 1.37 & 1.37 & 1.35 \\
\hline $\mathrm{N}_{8}-\mathrm{C}_{1}$ & 1.44 & 1.44 & 1.45 & 1.45 & 1.45 \\
\hline $\mathrm{N}_{8}-\mathrm{C}_{7}$ & 1.35 & 1.36 & 1.35 & 1.37 & 1.35 \\
\hline $\mathrm{N}_{2}-\mathrm{C}_{10}$ & - & 1.45 & 1.44 & 1.46 & 1.44 \\
\hline $\mathrm{N}_{4}-\mathrm{C}_{9}$ & - & 1.47 & 1.47 & 1.46 & 1.44 \\
\hline $\mathrm{N}_{6}-\mathrm{C}_{8}$ & - & - & - & 1.44 & - \\
\hline $\mathrm{N}_{8}-\mathrm{C}_{11}$ & - & - & - & 1.45 & - \\
\hline
\end{tabular}

Таблица Д9 - Значение углов в градусах для гликолурила 1 и его N-фенил-, арильных производных 17-19

\begin{tabular}{|c|c|c|c|c|c|}
\hline Углы, град & 1 & $17 \mathbf{a}$ & $17 \mathrm{~b}$ & 18 & 19 \\
\hline $\mathrm{C}_{1}-\mathrm{N}_{2}-\mathrm{C}_{3}$ & 112.6 & 112.5 & 112.6 & 112.1 & 111.8 \\
\hline $\mathrm{C}_{3}-\mathrm{N}_{4}-\mathrm{C}_{5}$ & 112.6 & 112.3 & 112.4 & 112.3 & 112.4 \\
\hline $\mathrm{C}_{5}-\mathrm{N}_{6}-\mathrm{C}_{7}$ & 112.6 & 111.9 & 112.1 & 112.6 & 113.0 \\
\hline $\mathrm{C}_{1}-\mathrm{N}_{8}-\mathrm{C}_{7}$ & 112.6 & 113.1 & 113.1 & 112.4 & 112.9 \\
\hline $\mathrm{N}_{2}-\mathrm{C}_{1}-\mathrm{C}_{5}$ & 102.8 & 103.5 & 103.6 & 103.4 & 103.0 \\
\hline $\mathrm{N}_{2}-\mathrm{C}_{1}-\mathrm{N}_{8}$ & 114.7 & 114.6 & 113.8 & 113.7 & 114.4 \\
\hline $\mathrm{N}_{2}-\mathrm{C}_{3}-\mathrm{N}_{4}$ & 109.1 & 109.2 & 108.5 & 107.9 & 109.3 \\
\hline $\mathrm{N}_{4}-\mathrm{C}_{5}-\mathrm{C}_{1}$ & 102.8 & 102.5 & 102.5 & 103.6 & 103.1 \\
\hline $\mathrm{N}_{4}-\mathrm{C}_{5}-\mathrm{N}_{6}$ & 114.7 & 114.4 & 114.9 & 114.2 & 114.1 \\
\hline $\mathrm{N}_{6}-\mathrm{C}_{5}-\mathrm{C}_{1}$ & 102.8 & 103.5 & 103.5 & 102.9 & 102.0 \\
\hline $\mathrm{N}_{6}-\mathrm{C}_{7}-\mathrm{N}_{8}$ & 109.1 & 108.1 & 108.4 & 108.1 & 108.8 \\
\hline $\mathrm{N}_{8}-\mathrm{C}_{1}-\mathrm{C}_{5}$ & 102.8 & 102.5 & 102.4 & 103.6 & 103.2 \\
\hline $\mathrm{O}_{1}-\mathrm{C}_{3}-\mathrm{N}_{4}$ & 125.5 & 125.0 & 126.1 & 126.1 & 124.8 \\
\hline $\mathrm{O}_{1}-\mathrm{C}_{3}-\mathrm{N}_{2}$ & 125.5 & 125.8 & 125.4 & 126.0 & 125.9 \\
\hline $\mathrm{O}_{2}-\mathrm{C}_{7}-\mathrm{N}_{8}$ & 125.5 & 125.6 & 126.7 & 126.0 & 125.7 \\
\hline $\mathrm{O}_{2}-\mathrm{C}_{7}-\mathrm{N}_{6}$ & 125.5 & 126.4 & 124.9 & 125.9 & 125.5 \\
\hline $\mathrm{C}_{1}-\mathrm{N}_{8}-\mathrm{C}_{11}$ & - & - & - & 121.7 & - \\
\hline $\mathrm{C}_{1}-\mathrm{N}_{2}-\mathrm{C}_{10}$ & - & 121.8 & 122.1 & 122.1 & 122.9 \\
\hline $\mathrm{C}_{3}-\mathrm{N}_{2}-\mathrm{C}_{10}$ & - & 125.7 & 124.9 & 121.3 & 122.3 \\
\hline $\mathrm{C}_{3}-\mathrm{N}_{4}-\mathrm{C}_{9}$ & - & 121.0 & 122.4 & 120.2 & 123.9 \\
\hline $\mathrm{C}_{5}-\mathrm{N}_{4}-\mathrm{C}_{9}$ & - & 126.2 & 125.1 & 122.7 & 123.5 \\
\hline $\mathrm{C}_{5}-\mathrm{N}_{6}-\mathrm{C}_{8}$ & - & - & - & 123.5 & - \\
\hline $\mathrm{C}_{7}-\mathrm{N}_{6}-\mathrm{C}_{8}$ & - & - & - & 122.5 & - \\
\hline $\mathrm{C}_{7}-\mathrm{N}_{8}-\mathrm{C}_{11}$ & - & - & - & 121.6 & - \\
\hline $\begin{array}{c}\text { Угол между } \\
\text { плоскостями }\end{array}$ & 124.1 & 122.1 & 118.8 & 124.2 & 11.3 \\
\hline
\end{tabular}

Таблица Д10 - Водородные связи в молекуле 20

\begin{tabular}{|c|c|c|c|}
\hline \multirow{2}{*}{ Связь } & \multicolumn{2}{|c|}{ Расстояние, $\AA$} & Угол, град \\
\cline { 2 - 4 } & D... & H... & D-Н... \\
\hline $\mathrm{N}_{4}-\mathrm{H} \ldots \mathrm{O}_{1}$ & $2.882(2)$ & 2.06 & 163 \\
\hline $\mathrm{N}_{6}-\mathrm{H} \ldots \mathrm{O}_{3}$ & $2.924(2)$ & 2.06 & 156 \\
\hline $\mathrm{N}_{8}-\mathrm{H} \ldots \mathrm{O}_{1}$ & $3.054(2)$ & 2.29 & 165 \\
\hline
\end{tabular}




\begin{tabular}{|c|c|c|c|}
\hline $\mathrm{O}_{4}-\mathrm{H} \ldots \mathrm{O}_{2}$ & $2.626(2)$ & 1.69 & 170 \\
\hline $\mathrm{O}_{\mathrm{W}}-\mathrm{H}_{\mathrm{W}} \ldots \mathrm{O}_{1}$ & $2.780(3)$ & 1.85 & 152 \\
\hline $\mathrm{O}_{\mathrm{W}}-\mathrm{H}_{\mathrm{W}} \ldots \mathrm{N}_{4}$ & $3.310(3)$ & 2.36 & 152 \\
\hline $\mathrm{C}_{5}-\mathrm{H} \ldots \mathrm{O}_{2}$ & $3.231(3)$ & 2.17 & 172 \\
\hline
\end{tabular}

Таблица Д11 - Значение длин связей для гликолурила 1 и его производных 20-24

\begin{tabular}{|c|c|c|c|c|c|c|}
\hline $\begin{array}{c}\text { Связи, } \\
\AA\end{array}$ & 1 & 20 & 21 & 22 & 23 & 24 \\
\hline $\mathrm{O}_{1}-\mathrm{C}_{3}$ & 1.24 & .24 & 1.22 & 1.20 & 1.20 & 1.19 \\
\hline $\mathrm{O}_{2}-\mathrm{C}_{7}$ & 1.24 & .24 & 1.22 & 1.21 & 1.21 & 1.19 \\
\hline $\mathrm{C}_{1}-\mathrm{C}_{5}$ & .56 & .56 & 1.56 & 1.53 & 1.57 & 1.55 \\
\hline $\mathrm{N}_{2}-\mathrm{C}_{1}$ & 1.44 & .45 & 1.45 & 1.45 & 1.47 & 1.48 \\
\hline $\mathrm{N}_{2}-\mathrm{C}_{3}$ & 1.35 & 1.35 & 1.36 & 1.40 & 1.43 & 1.41 \\
\hline $\mathrm{N}_{4}-\mathrm{C}_{3}$ & $\begin{array}{ll}1 & 35 \\
\end{array}$ & 1.35 & 1.39 & 1.40 & 1.34 & 1.40 \\
\hline $\mathrm{N}_{4}-\mathrm{C}_{5}$ & 1.44 & 1.44 & 1.45 & 1.48 & 1.44 & 1.45 \\
\hline $\mathrm{N}_{6}-\mathrm{C}_{5}$ & 1.44 & 1.44 & 1.45 & 1.45 & 1.46 & 1.47 \\
\hline $\mathrm{N}_{6}-\mathrm{C}_{7}$ & 35 & 1.35 & 1.36 & 1.41 & 1.41 & 1.42 \\
\hline $\mathrm{N}_{8}-\mathrm{C}_{1}$ & 1.44 & 1.44 & 1.45 & 1.47 & 1.44 & 1.46 \\
\hline $\mathrm{N}_{8}-\mathrm{C}_{7}$ & 1.35 & 1.36 & 1.36 & 1.39 & 1.35 & 1.41 \\
\hline $\mathrm{N}_{2}-\mathrm{C}_{10}$ & - & 1.46 & 1.44 & 1.42 & 1.36 & 1.40 \\
\hline $\mathrm{N}_{4}-\mathrm{C}_{9}$ & - & - & 1.43 & 1.40 & - & 1.44 \\
\hline $\mathrm{N}_{6}-\mathrm{C}_{8}$ & - & - & 1.44 & 1.42 & 1.39 & 1.40 \\
\hline $\mathrm{N}_{8}-\mathrm{C}_{11}$ & - & - & 1.43 & 1.41 & 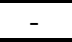 & 1.43 \\
\hline
\end{tabular}

Таблица Д12 - Значение углов для гликолурила 1 и его производных 20-24

\begin{tabular}{|c|c|c|c|c|c|c|}
\hline Углы, град & $\mathbf{1}$ & $\mathbf{2 0}$ & $\mathbf{2 1}$ & $\mathbf{2 2}$ & $\mathbf{2 3}$ & $\mathbf{2 4}$ \\
\hline $\mathrm{C}_{1}-\mathrm{N}_{2}-\mathrm{C}_{3}$ & 112.6 & 111.9 & 113.7 & 111.0 & 114.2 & 114.7 \\
\hline $\mathrm{C}_{3}-\mathrm{N}_{4}-\mathrm{C}_{5}$ & 112.6 & 112.5 & 111.7 & 12.0 & 116.0 & 112.3 \\
\hline $\mathrm{C}_{5}-\mathrm{N}_{6}-\mathrm{C}_{7}$ & 112.6 & 112.8 & - & 110.8 & 114.9 & 114.7 \\
\hline $\mathrm{C}_{1}-\mathrm{N}_{8}-\mathrm{C}_{7}$ & 112.6 & 111.9 & - & 112.0 & 104.1 & 112.1 \\
\hline $\mathrm{N}_{2}-\mathrm{C}_{1}-\mathrm{C}_{5}$ & 102.8 & 102.8 & - & 104.7 & 100.2 & 99.8 \\
\hline $\mathrm{N}_{2}-\mathrm{C}_{1}-\mathrm{N}_{8}$ & 114.7 & - & - & 111.4 & - & 113.0 \\
\hline $\mathrm{N}_{2}-\mathrm{C}_{3}-\mathrm{N}_{4}$ & 109.1 & 109.3 & 108.0 & 106.7 & 104.8 & 105.1 \\
\hline $\mathrm{N}_{4}-\mathrm{C}_{5}-\mathrm{C}_{1}$ & 102.8 & 103.1 & 103.7 & 101.3 & 104.3 & 105.7 \\
\hline $\mathrm{N}_{4}-\mathrm{C}_{5}-\mathrm{N}_{6}$ & 114.7 & 114.4 & 112.9 & 113.0 & - & 111.4 \\
\hline $\mathrm{N}_{6}-\mathrm{C}_{5}-\mathrm{C}_{1}$ & 102.8 & 102.3 & 102.7 & 104.2 & 100.1 & 100.1 \\
\hline $\mathrm{N}_{6}-\mathrm{C}_{7}-\mathrm{N}_{8}$ & 109.1 & 108.9 & - & 106.5 & 105.0 & 104.6 \\
\hline $\mathrm{N}_{8}-\mathrm{C}_{1}-\mathrm{C}_{5}$ & 102.8 & 103.3 & - & 101.6 & 100.1 & 105.4 \\
\hline $\mathrm{O}_{1}-\mathrm{C}_{3}-\mathrm{N}_{4}$ & 125.5 & 125.4 & 125.5 & 127.0 & 129.4 & 128.3 \\
\hline $\mathrm{O}_{1}-\mathrm{C}_{3}-\mathrm{N}_{2}$ & 125.5 & 125.3 & 126.4 & 126.3 & 125.8 & 126.6 \\
\hline $\mathrm{O}_{2}-\mathrm{C}_{7}-\mathrm{N}_{8}$ & 125.5 & 125.3 & 124.6 & 127.3 & 127.9 & 127.9 \\
\hline $\mathrm{O}_{2}-\mathrm{C}_{7}-\mathrm{N}_{6}$ & 125.5 & 125.7 & 126.8 & 126.1 & 127.2 & 127.5 \\
\hline $\mathrm{C}_{1}-\mathrm{N}_{8}-\mathrm{C}_{11}$ & - & - & - & 120.0 & - & 121.2 \\
\hline $\mathrm{C}_{1}-\mathrm{N}_{2}-\mathrm{C}_{10}$ & - & 123.1 & 122.7 & 121.0 & 120.6 & 120.1 \\
\hline $\mathrm{C}_{3}-\mathrm{N}_{2}-\mathrm{C}_{10}$ & - & 122.5 & 123.4 & 125.2 & 125.2 & 121.1 \\
\hline $\mathrm{C}_{3}-\mathrm{N}_{4}-\mathrm{C}_{9}$ & - & - & 120.3 & 126.3 & - & 123.5 \\
\hline $\mathrm{C}_{5}-\mathrm{N}_{4}-\mathrm{C}_{9}$ & - & - & 124.8 & 121.7 & - & 119.3 \\
\hline $\mathrm{C}_{5}-\mathrm{N}_{6}-\mathrm{C}_{8}$ & - & - & - & 119.5 & 117.2 & 119.6 \\
\hline $\mathrm{C}_{7}-\mathrm{N}_{6}-\mathrm{C}_{8}$ & - & - & - & 125.9 & 126.2 & 122.0 \\
\hline $\mathrm{C}_{7}-\mathrm{N}_{8}-\mathrm{C}_{11}$ & - & - & - & 127.7 & - & 124.4 \\
\hline У гол между $_{\text {плоскостями }}$ & 124.1 & 119.1 & 123.2 & 115.8 & 117.9 & 115.0 \\
лоск $_{1}$ & & & & & \\
\hline
\end{tabular}

Таблица Д13 - Геометрия водородных связей 25

\begin{tabular}{|c|c|c|c|c|}
\hline \multirow{2}{*}{ Связь } & \multicolumn{3}{|c|}{ Расстояние, $\AA$} & Угол, град \\
\cline { 2 - 5 } & $\mathrm{D}-\mathrm{H}$ & $\mathrm{H} \ldots \mathrm{A}$ & $\mathrm{D} \ldots \mathrm{A}$ & D-Н...А \\
\hline $\mathrm{N}_{1}-\mathrm{H}_{1 \mathrm{a}} \ldots \mathrm{O}_{3}{ }^{\mathrm{i}}$ & $0.90(3)$ & $2.22(3)$ & $3.098(3)$ & $164 .(3)$ \\
\hline $\mathrm{N}_{1}-\mathrm{H}_{1 \mathrm{~b}} \ldots \mathrm{O}_{7} 7^{\mathrm{ii}}$ & $0.89(3)$ & $2.15(3)$ & $3.039(3)$ & $170 .(3)$ \\
\hline $\mathrm{N}_{2}-\mathrm{H}_{2} \ldots \mathrm{O}_{7}$ iii & $0.86(3)$ & $2.01(3)$ & $2.833(3)$ & $159 .(3)$ \\
\hline $\mathrm{N}_{4}-\mathrm{H}_{4} \ldots \mathrm{N}_{1}{ }^{\mathrm{iv}}$ & $0.92(3)$ & $2.09(3)$ & $3.005(3)$ & $177 .(3)$ \\
\hline $\mathrm{N}_{5}-\mathrm{H}_{5} \ldots \mathrm{O}_{3}{ }^{\mathrm{i}}$ & $0.93(3)$ & $2.25(3)$ & $3.153(3)$ & $165 .(3)$ \\
\hline $\mathrm{N}_{5}-\mathrm{H}_{5} \ldots \mathrm{O}_{7}{ }^{\mathrm{ii}}$ & $0.94(3)$ & $2.40(3)$ & $3.280(3)$ & $155 .(3)$ \\
\hline $\mathrm{N}_{6}-\mathrm{H}_{6} \ldots \mathrm{N}_{5}{ }^{\mathrm{v}}$ & $0.88(3)$ & $2.19(3)$ & $3.066(3)$ & $177 .(3)$ \\
\hline $\mathrm{N}_{8}-\mathrm{H}_{8} \ldots \mathrm{O}_{3}{ }^{\mathrm{vi}}$ & $0.85(3)$ & $2.49(4)$ & $3.236(3)$ & $146 .(3)$ \\
\hline
\end{tabular}

Таблица Д14 - Система водородных связей молекулы 26

\begin{tabular}{|c|c|c|c|c|}
\hline \multirow{2}{*}{ Связь } & \multicolumn{3}{|c|}{ Расстояние, $\AA$} & $\begin{array}{c}\text { Угол, } \\
\text { град }\end{array}$ \\
\cline { 2 - 5 } & D-H & H... & D... A & D-H... \\
\hline $\mathrm{N}_{1}-\mathrm{H}_{1} \ldots \mathrm{O}_{2}{ }^{\mathrm{i}}$ & 0.86 & 2.15 & $2.963(3)$ & 158 \\
\hline $\mathrm{N}_{2}-\mathrm{H}_{2} \ldots \mathrm{O}_{7}{ }^{\mathrm{ii}}$ & 0.86 & 2.10 & $2.927(3)$ & 162 \\
\hline $\mathrm{N}_{3}-\mathrm{H}_{3} \ldots \mathrm{O}_{1}{ }^{\mathrm{iii}}$ & 0.86 & 2.11 & $2.937(3)$ & 161 \\
\hline $\mathrm{N}_{4}-\mathrm{H}_{4} \ldots \mathrm{O}_{8 \mathrm{i}}{ }^{\mathrm{v}}$ & 0.86 & 2.21 & $2.879(3)$ & 134 \\
\hline $\mathrm{N}_{5}-\mathrm{H}_{5} \ldots \mathrm{O}_{1 \mathrm{i}}{ }^{\mathrm{v}}$ & 0.86 & 2.22 & $2.870(3)$ & 133 \\
\hline $\mathrm{N}_{6}-\mathrm{H}_{6} \ldots \mathrm{O}_{7}{ }^{\mathrm{v}}$ & 0.86 & 2.09 & $2.925(3)$ & 164 \\
\hline $\mathrm{N}_{7}-\mathrm{H}_{7} \ldots \mathrm{O}_{2}{ }^{\mathrm{ii}}$ & 0.86 & 2.16 & $2.973(3)$ & 158 \\
\hline
\end{tabular}




\begin{tabular}{|c|c|c|c|c|}
\hline \multirow{2}{*}{ Связь } & \multicolumn{3}{|c|}{ Расстояние, $\AA$} & $\begin{array}{c}\text { Угол, } \\
\text { град }\end{array}$ \\
\cline { 2 - 5 } & D-H & H..A & D... A & D-H... \\
\hline $\mathrm{N}_{8}-\mathrm{H}_{8} \ldots \mathrm{O}_{8 \mathrm{v}}{ }^{\mathrm{i}}$ & 0.86 & 2.12 & $2.963(3)$ & 165 \\
\hline $\mathrm{C}_{16}-\mathrm{H}_{16} \ldots \mathrm{O}_{3}{ }^{\mathrm{vii}}$ & 0.96 & 2.51 & $3.087(5)$ & 119 \\
\hline $\mathrm{C}_{10}-\mathrm{H}_{10} \ldots \mathrm{O}_{11}{ }^{\mathrm{v}}$ & 0.96 & 2.32 & $3.162(5)$ & 146 \\
\hline
\end{tabular}

Таблица Д15 - Значение длин связей для гликолурила 1 и его производных 25-32

\begin{tabular}{|c|r|r|r|r|r|r|r|r|r|}
\hline Связи, $\AA$ & $\mathbf{1}$ & $\mathbf{2 5}$ & $\mathbf{2 6}$ & $\mathbf{2 7}$ & $\mathbf{2 8}$ & $\mathbf{2 9}$ & $\mathbf{3 0}$ & $\mathbf{3 1}$ & $\mathbf{3 2}$ \\
\hline $\mathrm{O}_{1}-\mathrm{C}_{3}$ & 1.24 & 1.23 & 1.22 & 1.24 & 1.22 & 1.22 & 1.21 & 1.20 & 1.21 \\
\hline $\mathrm{O}_{2}-\mathrm{C}_{7}$ & 1.24 & 1.23 & 1.23 & 1.24 & 1.22 & 1.24 & 1.22 & 1.21 & 1.21 \\
\hline $\mathrm{C}_{1}-\mathrm{C}_{5}$ & 1.56 & 1.61 & 1.59 & 1.58 & 1.57 & 1.57 & 1.57 & 1.57 & 1.55 \\
\hline $\mathrm{N}_{2}-\mathrm{C}_{1}$ & 1.44 & 1.44 & 1.43 & 1.44 & 1.44 & 1.50 & 1.50 & 1.47 & 1.44 \\
\hline $\mathrm{N}_{2}-\mathrm{C}_{3}$ & 1.35 & 1.35 & 1.36 & 1.34 & 1.34 & 1.40 & 1.40 & 1.40 & 1.37 \\
\hline $\mathrm{N}_{4}-\mathrm{C}_{3}$ & 1.35 & 1.36 & 1.36 & 1.34 & 1.44 & 1.34 & 1.35 & 1.36 & 1.38 \\
\hline $\mathrm{N}_{4}-\mathrm{C}_{5}$ & 1.44 & 1.44 & 1.44 & 1.44 & 1.44 & 1.48 & 1.46 & 1.45 & 1.46 \\
\hline $\mathrm{N}_{6}-\mathrm{C}_{5}$ & 1.44 & 1.45 & 1.44 & 1.44 & 1.44 & 1.46 & 1.46 & 1.45 & 1.45 \\
\hline $\mathrm{N}_{6}-\mathrm{C}_{7}$ & 1.35 & 1.35 & 1.35 & 1.34 & 1.44 & 1.36 & 1.35 & 1.34 & 1.35 \\
\hline $\mathrm{N}_{8}-\mathrm{C}_{1}$ & 1.44 & 1.44 & 1.44 & 1.44 & 1.44 & 1.43 & 1.46 & 1.49 & 1.48 \\
\hline $\mathrm{N}_{8}-\mathrm{C}_{7}$ & 1.35 & 1.36 & 1.36 & 1.34 & 1.34 & 1.35 & 1.41 & 1.40 & 1.40 \\
\hline $\mathrm{N}_{2}-\mathrm{C}_{10}$ & - & - & - & - & 1.43 & 1.37 & 1.38 & 1.43 & - \\
\hline $\mathrm{N}_{4}-\mathrm{C}_{9}$ & - & - & - & - & - & 1.45 & 1.45 & 1.46 & 1.44 \\
\hline $\mathrm{N}_{6}-\mathrm{C}_{8}$ & - & - & - & - & - & 1.45 & 1.44 & 1.46 & 1.46 \\
\hline $\mathrm{N}_{8}-\mathrm{C}_{11}$ & - & - & - & - & 1.43 & - & 1.41 & 1.40 & 1.38 \\
\hline & & & & & & & \\
\hline
\end{tabular}

Таблица Д16 - Значение углов для гликолурила 1 и его производных 25-32

\begin{tabular}{|c|c|c|c|c|c|c|c|c|c|}
\hline Углы, град & 1 & 25 & 26 & 27 & 28 & 29 & 30 & 31 & 32 \\
\hline $\mathrm{C}_{1}-\mathrm{N}_{2}-\mathrm{C}_{3}$ & 112.6 & 113.8 & 113.5 & 113.9 & 113.7 & 112.1 & 111.6 & 111.8 & 111.7 \\
\hline $\mathrm{C}_{3}-\mathrm{N}_{4}-\mathrm{C}_{5}$ & 112.6 & 112.8 & 112.7 & 113.9 & 113.0 & 113.3 & 113.5 & 114.0 & 111.2 \\
\hline $\mathrm{C}_{5}-\mathrm{N}_{6}-\mathrm{C}_{7}$ & 112.6 & 113.8 & 113.0 & 113.9 & - & 111.6 & 114.1 & 114.0 & 113.5 \\
\hline $\mathrm{C}_{1}-\mathrm{N}_{8}-\mathrm{C}_{7}$ & 112.6 & 113.1 & 113.4 & 113.9 & - & 112.0 & 110.4 & 111.1 & 111.3 \\
\hline $\mathrm{N}_{2}-\mathrm{C}_{1}-\mathrm{C}_{5}$ & 102.8 & 100.9 & 102.3 & 101.8 & 102.0 & 100.5 & 100.7 & 102.7 & 102.7 \\
\hline $\mathrm{N}_{2}-\mathrm{C}_{1}-\mathrm{N}_{8}$ & \begin{tabular}{|l|}
114.7 \\
\end{tabular} & 114.7 & 116.1 & 112.4 & 112.2 & 113.3 & 111.4 & 110.2 & 112.6 \\
\hline $\mathrm{N}_{2}-\mathrm{C}_{3}-\mathrm{N}_{4}$ & 109.1 & 108.9 & 108.2 & 108.4 & 108.6 & 107.6 & 107.8 & 107.3 & 108.1 \\
\hline $\mathrm{N}_{4}-\mathrm{C}_{5}-\mathrm{C}_{1}$ & 102.8 & 102.6 & 102.2 & 101.8 & 102.6 & 102.3 & 102.9 & 102.0 & 101.8 \\
\hline $\mathrm{N}_{4}-\mathrm{C}_{5}-\mathrm{N}_{6}$ & \begin{tabular}{|l|}
114.7 \\
\end{tabular} & 114.8 & 115.3 & 112.4 & 111.8 & 112.4 & 111.5 & 112.6 & 112.7 \\
\hline $\mathrm{N}_{6}-\mathrm{C}_{5}-\mathrm{C}_{1}$ & 102.8 & 101.2 & 102.8 & 101.8 & - & 100.4 & 101.7 & 102.9 & 102.4 \\
\hline $\mathrm{N}_{6}-\mathrm{C}_{7}-\mathrm{N}_{8}$ & 109.1 & 108.9 & 109.0 & 108.4 & - & 108.7 & 107.6 & 107.8 & 106.9 \\
\hline $\mathrm{N}_{8}-\mathrm{C}_{1}-\mathrm{C}_{5}$ & \begin{tabular}{|l|l|}
102.8 \\
\end{tabular} & 102.1 & 101.6 & 101.8 & - & 102.7 & 102.7 & 101.1 & 101.2 \\
\hline $\mathrm{O}_{1}-\mathrm{C}_{3}-\mathrm{N}_{4}$ & 125.5 & 125.9 & 125.6 & 125.8 & 125.1 & 125.8 & 125.2 & 127.1 & 125.9 \\
\hline $\mathrm{O}_{1}-\mathrm{C}_{3}-\mathrm{N}_{2}$ & 125.5 & 125.2 & 126.2 & 125.8 & 126.4 & 126.5 & 127.0 & 125.6 & 126.0 \\
\hline $\mathrm{O}_{2}-\mathrm{C}_{7}-\mathrm{N}_{8}$ & 125.5 & 126.0 & 125.8 & 125.8 & - & 124.8 & 126.6 & 125.7 & 126.2 \\
\hline $\mathrm{O}_{2}-\mathrm{C}_{7}-\mathrm{N}_{6}$ & 125.5 & 125.1 & 125.2 & 125.8 & - & 126.6 & 125.8 & 126.5 & 126.9 \\
\hline $\mathrm{C}_{1}-\mathrm{N}_{8}-\mathrm{C}_{11}$ & - & - & - & - & - & - & 125.7 & 122.0 & 121.4 \\
\hline $\mathrm{C}_{1}-\mathrm{N}_{2}-\mathrm{C}_{10}$ & - & - & - & - & 124.7 & 119.8 & 122.2 & 126.4 & - \\
\hline $\mathrm{C}_{3}-\mathrm{N}_{2}-\mathrm{C}_{10}$ & - & - & - & - & 121.5 & 128.1 & 126.1 & 121.6 & - \\
\hline $\mathrm{C}_{3}-\mathrm{N}_{4}-\mathrm{C}_{9}$ & - & - & - & - & - & 123.3 & 121.0 & 121.1 & 118.9 \\
\hline $\mathrm{C}_{5}-\mathrm{N}_{4}-\mathrm{C}_{9}$ & - & - & - & - & - & 123.1 & 125.4 & 124.1 & 124.6 \\
\hline $\mathrm{C}_{5}-\mathrm{N}_{6}-\mathrm{C}_{8}$ & - & - & - & - & - & 124.0 & 124.9 & 124.4 & 124.8 \\
\hline $\mathrm{C}_{7}-\mathrm{N}_{6}-\mathrm{C}_{8}$ & - & - & - & - & - & 120.5 & 120.4 & 121.6 & 121.1 \\
\hline $\mathrm{C}_{7}-\mathrm{N}_{8}-\mathrm{C}_{11}$ & - & - & - & - & - & - & 123.4 & 126.9 & 127.2 \\
\hline $\begin{array}{l}\text { Угол между плоско- } \\
\text { стями }\end{array}$ & 124.1 & 123.6 & 112.5 & 130.0 & 118.6 & 117.6 & 113.5 & 118.4 & 116.3 \\
\hline
\end{tabular}


Таблица Д17 - Значение длин связей для гликолурила 1 и его производных 33-39. Сравнение длин связей для соединений 1, 33-39 отображены в графике на рисунке 61 .

\begin{tabular}{|c|c|c|c|c|c|c|c|c|}
\hline Связи, $\AA$ & $\mathbf{1}$ & $\mathbf{3 3}$ & $\mathbf{3 4}$ & $\mathbf{3 5}$ & $\mathbf{3 6}$ & $\mathbf{3 7}$ & $\mathbf{3 8}$ & $\mathbf{3 9}$ \\
\hline $\mathrm{O}_{1}-\mathrm{C}_{3}$ & 1.24 & 1.23 & 1.23 & 1.23 & 1.22 & 1.23 & 1.23 & 1.21 \\
\hline $\mathrm{O}_{2}-\mathrm{C}_{7}$ & 1.24 & 1.22 & 1.22 & 1.24 & 1.23 & 1.23 & 1.24 & 1.21 \\
\hline $\mathrm{C}_{1}-\mathrm{C}_{5}$ & 1.56 & 1.59 & 1.59 & 1.59 & 1.61 & 1.60 & 1.60 & 1.61 \\
\hline $\mathrm{N}_{2}-\mathrm{C}_{1}$ & 1.44 & 1.46 & 1.44 & 1.45 & 1.44 & 1.45 & 1.46 & 1.45 \\
\hline $\mathrm{N}_{2}-\mathrm{C}_{3}$ & 1.35 & 1.34 & 1.37 & 1.38 & 1.35 & 1.37 & 1.37 & 1.39 \\
\hline $\mathrm{N}_{4}-\mathrm{C}_{3}$ & 1.35 & 1.36 & 1.36 & 1.35 & 1.37 & 1.36 & 1.38 & 1.39 \\
\hline $\mathrm{N}_{4}-\mathrm{C}_{5}$ & 1.44 & 1.44 & 1.45 & 1.47 & 1.47 & 1.46 & 1.44 & 1.47 \\
\hline $\mathrm{N}_{6}-\mathrm{C}_{5}$ & 1.44 & 1.45 & 1.45 & 1.45 & 1.46 & 1.46 & 1.46 & 1.45 \\
\hline $\mathrm{N}_{6}-\mathrm{C}_{7}$ & 1.35 & 1.34 & 1.36 & 1.37 & 1.38 & 1.38 & 1.36 & 1.39 \\
\hline $\mathrm{N}_{8}-\mathrm{C}_{1}$ & 1.44 & 1.44 & 1.46 & 1.46 & 1.45 & 1.46 & 1.45 & 1.47 \\
\hline $\mathrm{N}_{8}-\mathrm{C}_{7}$ & 1.35 & 1.36 & 1.36 & 1.36 & 1.36 & 1.36 & 1.37 & 1.39 \\
\hline $\mathrm{N}_{2}-\mathrm{C}_{10}$ & - & - & 1.45 & 1.45 & - & - & 1.45 & 1.69 \\
\hline $\mathrm{N}_{4}-\mathrm{C}_{9}$ & - & - & 1.45 & 1.46 & 1.46 & 1.46 & 1.46 & 1.70 \\
\hline $\mathrm{N}_{6}-\mathrm{C}_{8}$ & - & - & 1.45 & - & 1.47 & 1.47 & - & 1.70 \\
\hline $\mathrm{N}_{8}-\mathrm{C}_{11}$ & - & - & 1.45 & - & - & - & 1.45 & 1.69 \\
\hline
\end{tabular}

Таблица Д18 - Значение углов для гликолурила 1 и его производных 33-39

\begin{tabular}{|c|c|c|c|c|c|c|c|c|}
\hline Углы, град & 1 & 33 & 34 & 35 & 36 & 37 & 38 & 39 \\
\hline $\mathrm{C}_{1}-\mathrm{N}_{2}-\mathrm{C}_{3}$ & 112.6 & 113.9 & 111.8 & 109.5 & 113.0 & 110.7 & 112.9 & 112.7 \\
\hline $\mathrm{C}_{3}-\mathrm{N}_{4}-\mathrm{C}_{5}$ & 112.6 & 112.8 & 112.6 & 112.6 & 100.9 & 112.4 & 110.7 & 114.0 \\
\hline $\mathrm{C}_{5}-\mathrm{N}_{6}-\mathrm{C}_{7}$ & 112.6 & 133.8 & 111.9 & 110.3 & 111.0 & 110.0 & 112.0 & 113.1 \\
\hline $\mathrm{C}_{1}-\mathrm{N}_{8}-\mathrm{C}_{7}$ & 112.6 & 112.9 & 112.6 & 112.5 & 113.4 & 112.5 & 111.5 & 113.6 \\
\hline $\mathrm{N}_{2}-\mathrm{C}_{1}-\mathrm{C}_{5}$ & 102.8 & 100.6 & 102.1 & 101.9 & \begin{tabular}{|l|}
101.9 \\
\end{tabular} & 102.0 & 100.4 & 100.7 \\
\hline $\mathrm{N}_{2}-\mathrm{C}_{1}-\mathrm{N}_{8}$ & 114.7 & 112.6 & 113.2 & 111.9 & 112.5 & 113.0 & 112.6 & 112.4 \\
\hline $\mathrm{N}_{2}-\mathrm{C}_{3}-\mathrm{N}_{4}$ & 109.1 & 108.9 & 109.0 & 108.9 & 109.6 & 109.1 & 107.9 & 105.5 \\
\hline $\mathrm{N}_{4}-\mathrm{C}_{5}-\mathrm{C}_{1}$ & 102.8 & 102.0 & \begin{tabular}{|l|}
101.1 \\
\end{tabular} & 99.6 & 100.9 & 99.8 & 101.6 & 99.5 \\
\hline $\mathrm{N}_{4}-\mathrm{C}_{5}-\mathrm{N}_{6}$ & 114.7 & 112.7 & 113.4 & 112.3 & 112.8 & 113.4 & 112.6 & 112.0 \\
\hline $\mathrm{N}_{6}-\mathrm{C}_{5}-\mathrm{C}_{1}$ & 102.8 & 100.5 & 102.0 & 101.6 & 101.9 & 102.0 & 100.4 & 100.7 \\
\hline $\mathrm{N}_{6}-\mathrm{C}_{7}-\mathrm{N}_{8}$ & 109.1 & 108.9 & 108.9 & 108.8 & 108.9 & 109.3 & 108.9 & 105.7 \\
\hline $\mathrm{N}_{8}-\mathrm{C}_{1}-\mathrm{C}_{5}$ & 102.8 & 102.2 & 100.9 & 99.9 & 100.9 & 100.0 & 101.5 & 99.9 \\
\hline $\mathrm{O}_{1}-\mathrm{C}_{3}-\mathrm{N}_{4}$ & 125.5 & 125.5 & 126.0 & 127.0 & 124.8 & 124.5 & 126.0 & 127.4 \\
\hline $\mathrm{O}_{1}-\mathrm{C}_{3}-\mathrm{N}_{2}$ & 125.5 & 125.6 & 125.0 & 124.1 & 125.8 & 126.4 & 126.0 & 127.1 \\
\hline $\mathrm{O}_{2}-\mathrm{C}_{7}-\mathrm{N}_{8}$ & 125.5 & 125.6 & 125.7 & 125.9 & 126.9 & 125.5 & 123.7 & 127.0 \\
\hline $\mathrm{O}_{2}-\mathrm{C}_{7}-\mathrm{N}_{6}$ & 125.5 & 125.5 & 125.4 & 125.3 & 124.2 & 125.2 & 127.4 & 127.3 \\
\hline $\mathrm{C}_{1}-\mathrm{N}_{8}-\mathrm{C}_{11}$ & - & - & 124.9 & - & - & - & 128.2 & 121.4 \\
\hline $\mathrm{C}_{1}-\mathrm{N}_{2}-\mathrm{C}_{10}$ & - & - & 125.1 & 119.0 & - & - & 123.1 & 118.9 \\
\hline $\mathrm{C}_{3}-\mathrm{N}_{2}-\mathrm{C}_{10}$ & - & - & 120.9 & 120.3 & - & - & 123.9 & 117.1 \\
\hline $\mathrm{C}_{3}-\mathrm{N}_{4}-\mathrm{C}_{9}$ & - & - & 121.9 & 124.1 & 118.8 & 121.0 & 119.1 & 117.7 \\
\hline $\mathrm{C}_{5}-\mathrm{N}_{4}-\mathrm{C}_{9}$ & - & - & 124.5 & 112.6 & \begin{tabular}{|l|}
123.4 \\
\end{tabular} & 126.1 & 119.5 & 122.1 \\
\hline $\mathrm{C}_{5}-\mathrm{N}_{6}-\mathrm{C}_{8}$ & - & - & 123.6 & - & 122.4 & 125.2 & - & 119.3 \\
\hline $\mathrm{C}_{7}-\mathrm{N}_{6}-\mathrm{C}_{8}$ & - & - & 120.7 & - & 119.2 & 120.2 & - & 117.1 \\
\hline $\mathrm{C}_{7}-\mathrm{N}_{8}-\mathrm{C}_{11}$ & - & - & 122.1 & - & - & - & 119.3 & 117.3 \\
\hline Угол между плоскостя- & 124. & 114. & 114. & 110. & 116. & 113. & 112. & 111. \\
\hline ми & 1 & 7 & 8 & 1 & 1 & 4 & 7 & 5 \\
\hline
\end{tabular}




\section{Рисунки}

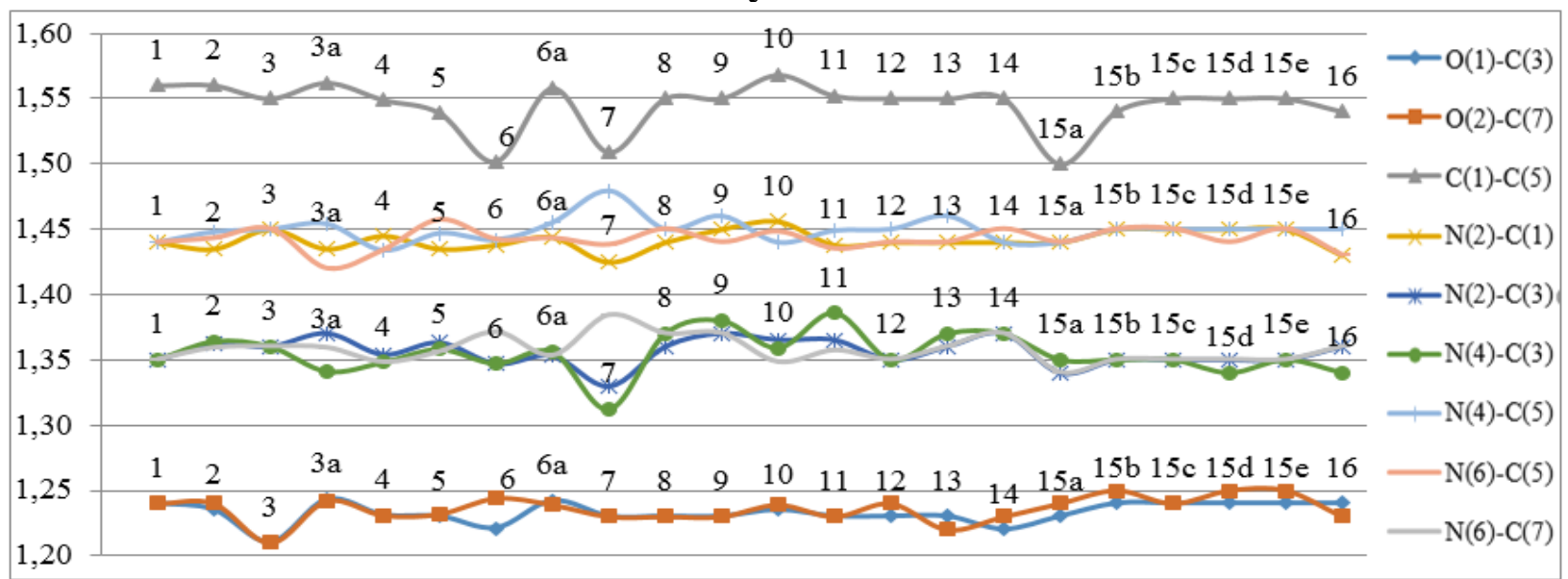

Рис. Д1. Сравнение длин связей (ангстрем, А̊) для гликолурила 1 и Nалкилзамещенных соединений 2-14, 16 и металлических комплексов 3a, 6а и 15a-e.

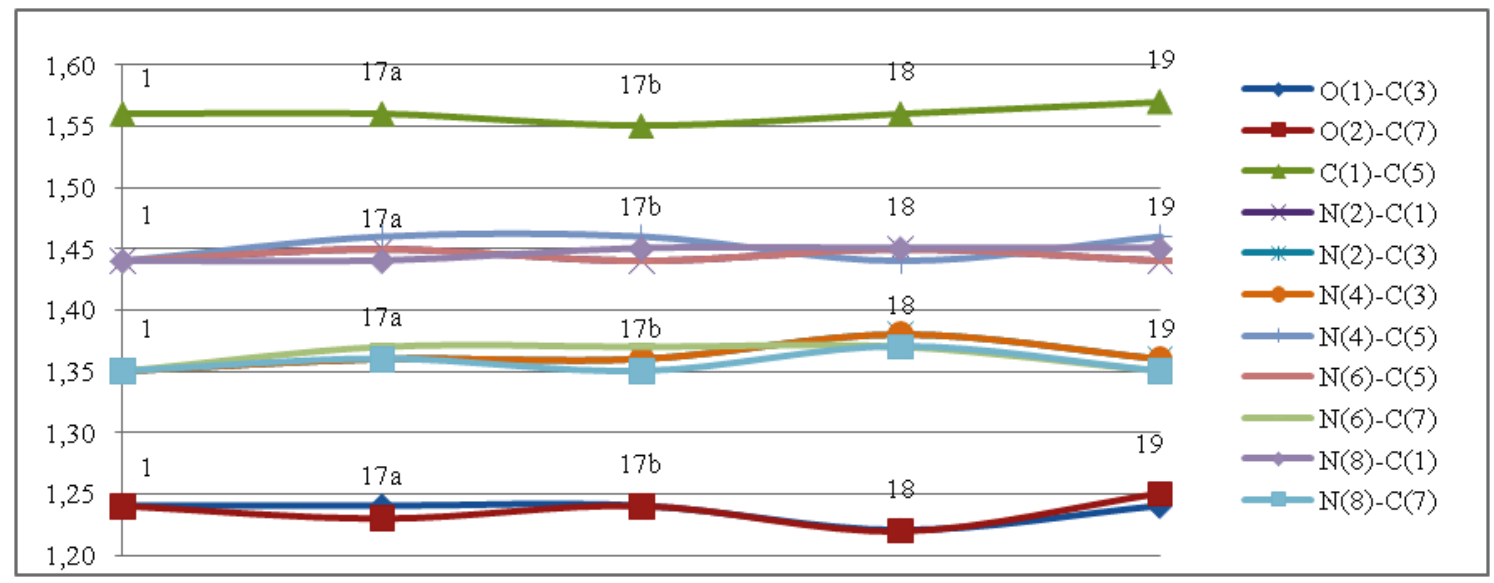

Рис. Д2. Сравнение длин связей гликолурила 1 с его производными 17-19 


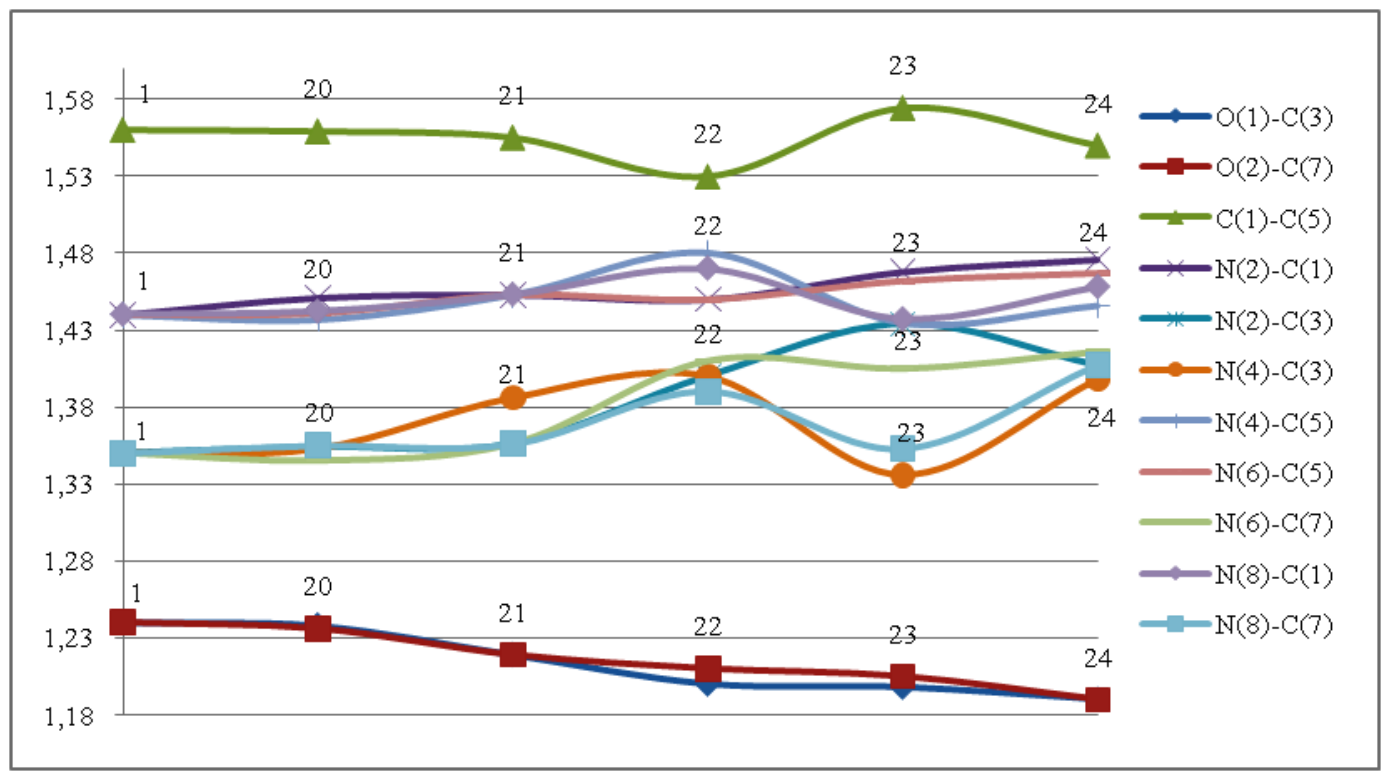

Рис. Д3. Сравнение длин связей гликолурила $\mathbf{1}$ с его производными 20-24

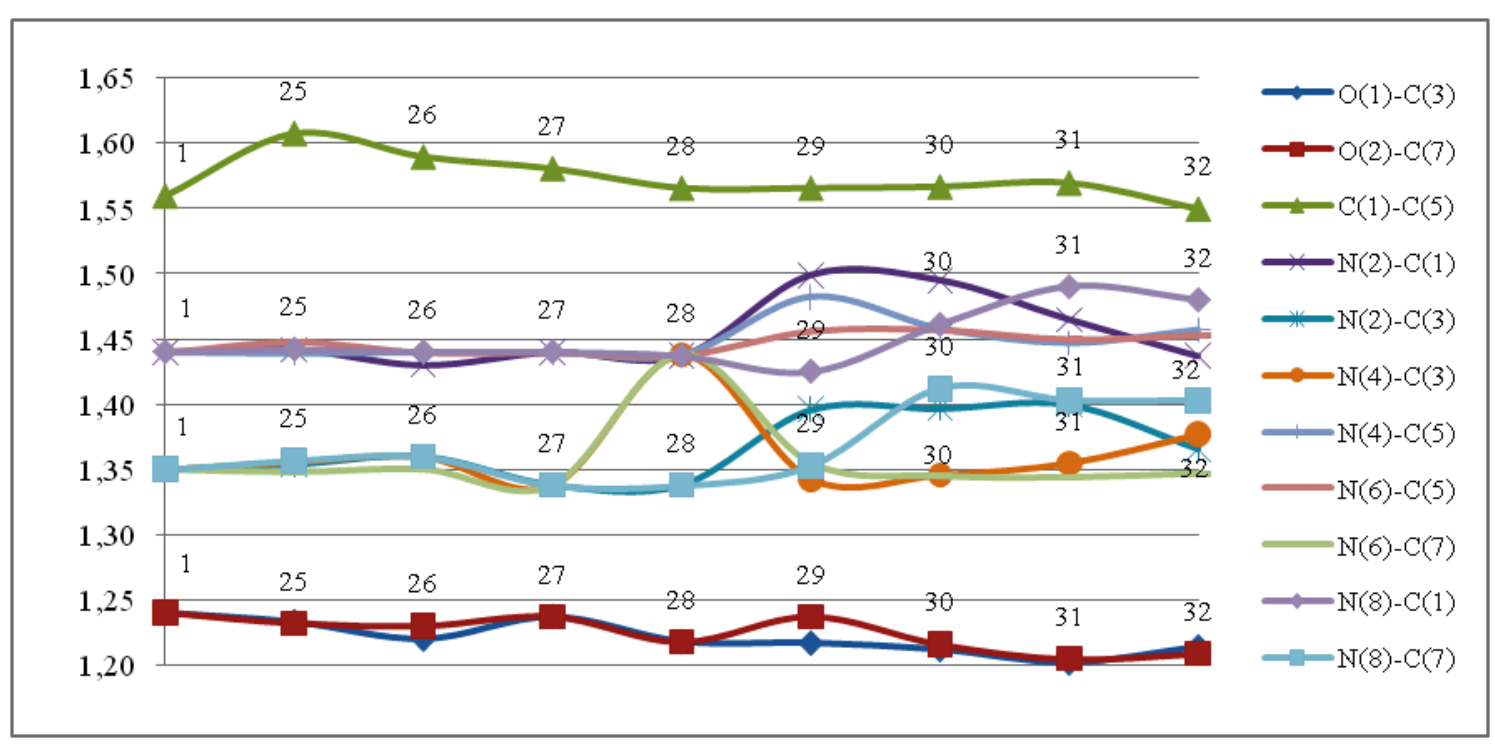

Рис. Д4. Сравнение длин связей гликолурила 1 с его производными 25-32 


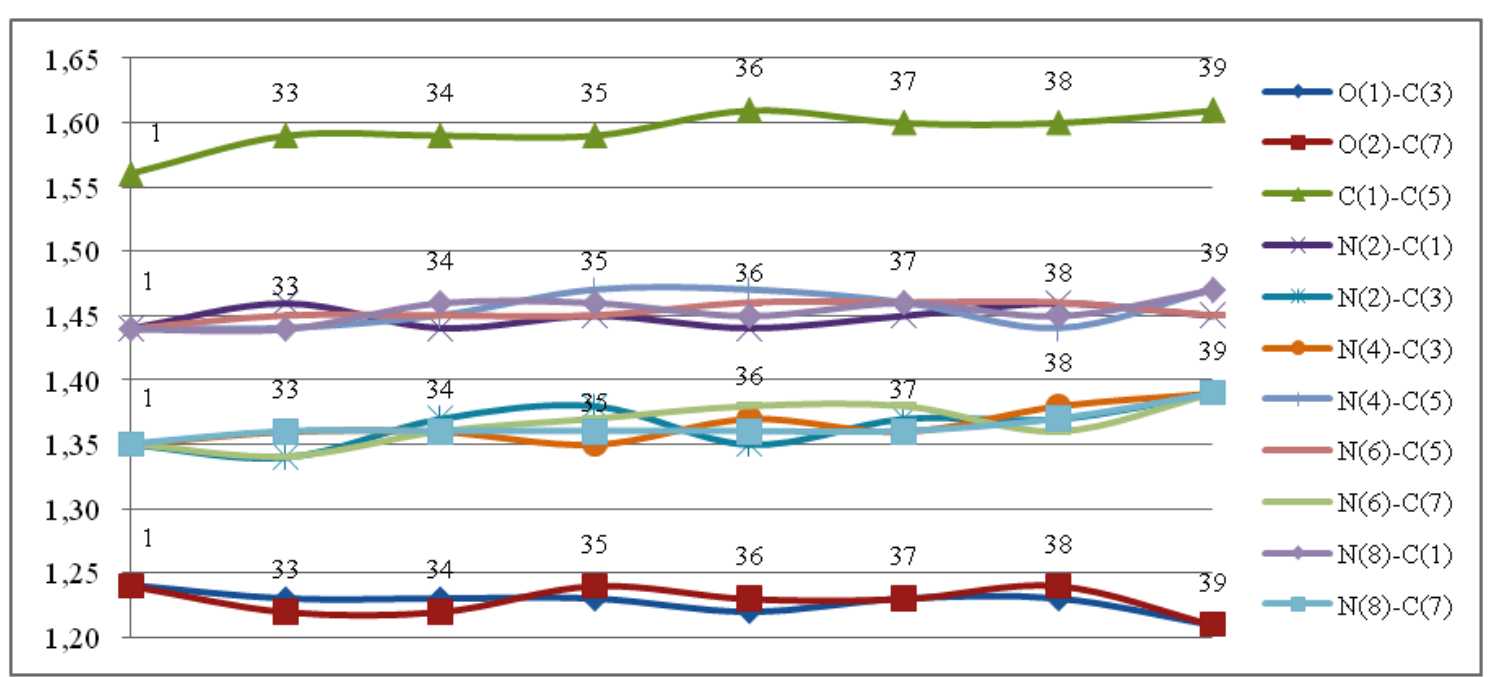

Рис. Д5. Сравнение длин связей гликолурила 1 с его производными 33-39 